\title{
Debugging Distributed Systems with Why-Across-Time Provenance
}

\author{
Michael Whittaker \\ UC Berkeley \\ mjwhittaker@berkeley.edu \\ Peter Alvaro \\ UC Santa Cruz \\ palvaro@ucsc.edu
}

\author{
Cristina Teodoropol \\ UC Berkeley \\ ct@berkeley.edu
}

\author{
Joseph M. Hellerstein \\ UC Berkeley \\ hellerstein@berkeley.edu
}

\begin{abstract}
Systematically reasoning about the fine-grained causes of events in a real-world distributed system is challenging. Causality, from the distributed systems literature, can be used to compute the causal history of an arbitrary event in a distributed system, but the event's causal history is an overapproximation of the true causes. Data provenance, from the database literature, precisely describes why a particular tuple appears in the output of a relational query, but data provenance is limited to the domain of static relational databases. In this paper, we present wat-provenance: a novel form of provenance that provides the benefits of causality and data provenance. Given an arbitrary state machine, watprovenance describes why the state machine produces a particular output when given a particular input. This enables system developers to reason about the causes of events in real-world distributed systems. We observe that automatically extracting the wat-provenance of a state machine is often infeasible. Fortunately, many distributed systems components have simple interfaces from which a developer can directly specify wat-provenance using a technique we call wat-provenance specifications. Leveraging the theoretical foundations of wat-provenance, we implement a prototype distributed debugging framework called Watermelon.
\end{abstract}

\footnotetext{
Permission to make digital or hard copies of all or part of this work for personal or classroom use is granted without fee provided that copies are not made or distributed for profit or commercial advantage and that copies bear this notice and the full citation on the first page. Copyrights for components of this work owned by others than ACM must be honored. Abstracting with credit is permitted. To copy otherwise, or republish, to post on servers or to redistribute to lists, requires prior specific permission and/or a fee. Request permissions from permissions@acm.org.

SoCC '18, October 11-13, 2018, Carlsbad, CA, USA

(C) 2018 Association for Computing Machinery.

ACM ISBN 978-1-4503-6011-1/18/10 . \$ \$15.00

https://doi.org/10.1145/3267809.3267839
}

\section{CCS CONCEPTS}

- Information systems $\rightarrow$ Data provenance; • Theory of computation $\rightarrow$ Data provenance; Formal languages and automata theory; • Software and its engineering $\rightarrow$ Software testing and debugging;

\section{KEYWORDS}

data provenance, state machines, distributed systems

ACM Reference Format:

Michael Whittaker, Cristina Teodoropol, Peter Alvaro, and Joseph M. Hellerstein. 2018. Debugging Distributed Systems with WhyAcross-Time Provenance. In Proceedings of ACM Symposium on Cloud Computing, Carlsbad, CA, USA, October 11-13, 2018 (SoCC '18), 14 pages.

https://doi.org/10.1145/3267809.3267839

\section{INTRODUCTION}

Debugging distributed systems is hard. Traditional debugging techniques are poorly suited to distributed systems in which bugs arise across multiple nodes connected by an unreliable network that can drop, duplicate, and reorder messages. Distributed debugging tools exist but are in their infancy. They help tame some of the complexities of distributed debugging, but have limited applicability to real-world distributed systems that are made up of a large number of complex components. Consequently, developers perform ad-hoc root cause analysis to find the source of a bug, stitching together the logs of multiple concurrently executing nodes.

Worse, existing formalisms are also inadequate to reason about systematically debugging real-world distributed systems. For example, consider causality [20]. Causality is a general-purpose formalism that specifies the causal history of a particular event in an arbitrary distributed system. However, causality is too general-purpose, as it fails to incorporate any semantics of the underlying distributed system [2]. As a consequence, the causal history of an event is an overapproximation of the cause of the event. It includes all the 
events that might have caused a particular event instead of the events that actually do cause it.

Alternatively, consider data provenance in the form of why-provenance $[5,7]$. Given a relational database, a query issued against the database, and a tuple in the output of the query, why-provenance explains why the output tuple was produced. That is, why-provenance produces the input tuples that, if passed through the relational operators of the query, would produce the output tuple in question. In contrast to causality, data provenance heavily incorporates the semantics of relational databases and queries to describe precisely the cause of a particular output. However, why-provenance makes the critical assumption that the underlying relational database is static. It cannot handle the time-varying nature of stateful distributed systems. Moreover, data provenance is limited to the domain of relational data and cannot easily be applied to other system components (e.g., load balancers, file systems, coordination services, etc.).

In short, causality lacks a notion of data dependence, and data provenance lacks a notion of time. In this paper, we present wat-provenance (why-across-time provenance): a novel form of data provenance that unifies ideas from the two. Wat-provenance generalizes why-provenance from the domain of relational queries issued against a static database to the domain of arbitrary time-varying state machines in a distributed system. More specifically, given a deterministic state machine, the state machine's sequence of inputs, and a particular input to the state machine, wat-provenance formalizes why the state machine produces the output that it does. This description is the set of subsequences of the input trace that are necessary and sufficient to generate the output in question. Borrowing from causality, wat-provenance can be applied to time-varying state machines. Borrowing from why-provenance, wat-provenance incorporates state machine semantics to avoid overapproximating provenance.

After we define wat-provenance, we turn to the matter of computing it. We observe that automatically extracting the wat-provenance of a state machine is often infeasible. Computing the wat-provenance of a state machine is tantamount to inferring the state machine's data dependencies using a complex code analysis of the state machine's source code. This source code can be both large and complex which makes this code analysis intractable. Though automatically extracting the wat-provenance of an arbitrary state machine is difficult, many distributed systems components are designed with simple and minimalistic APIs. We can take advantage of this observation and sidestep the complexity of extracting the wat-provenance from the implementation of a state machine and instead specify the wat-provenance from the interface of a state machine. To this end, we propose watprovenance specifications: functions that directly encode the wat-provenance of a state machine using its interface instead of its implementation. We describe the provenance specifications of a number of widely used distributed systems components (e.g., Redis, Amazon S3, HDFS, Zookeeper) and find that in practice, they are often straightforward to implement.

Next, we present Watermelon: a prototype distributed debugging framework that leverages the formal foundations of wat-provenance and wat-provenance specifications. Watermelon includes a mechanism for developers to write watprovenance specifications that are executed against the input traces of the components in a distributed system. We use Watermelon to measure the complexity of writing watprovenance specifications and also compare Watermelon to existing distributed debugging techniques.

This paper presents the following contributions:

- We define wat-provenance: a formalism that extends notions of data provenance to the realm of state machines in a distributed system.

- We present wat-provenance specifications: a mechanism to compute the wat-provenance of distributed system components. We also describe a set of watprovenance specifications for a number of widely used distributed systems components, illustrating that watprovenance specifications can be straightforward to write.

- We implement a prototype distributed debugger called Watermelon that leverages the theoretical foundations of wat-provenance. We demonstrate that Watermelon makes it easier to identify the precise causes of events in a distributed system compared to existing debugging techniques.

\section{BACKGROUND}

\subsection{Causality}

As described by Lamport in [20], time is fundamental to our understanding of how events are ordered. It is clear that if an event occurs at 6:42, then it happens before another event that occurs at 6:45. Unfortunately, accurately measuring time in a distributed system is infeasible [26, 29, 30]. Clocks on different servers within a distributed system drift apart, so servers cannot agree on a single global notion of time, and thus they cannot agree on a single global total order of events that respects the real time ordering of events. However, as Lamport showed in [20], it is possible for servers to agree on a global partial order of events that respects the global passage of time. This partial ordering of events also dictates which events can causally affect each other.

To make this partial ordering and notion of causality precise, we consider a set of single-threaded servers that communicate over the network. Every server $a$ serially executes 
Debugging Distributed Systems with Why-Across-Time Provenance

a sequence of events $a_{1}, a_{2}, a_{3}, \ldots$ where each event $a_{i}$ represents the action of server $a$ either (1) performing local computation, (2) sending a message to another server, or (3) receiving a message from another server.

The happens before relation $\rightarrow$ on events is the smallest transitive relation such that (1) if $a_{i}, a_{j}$ are two distinct events within the same process, then $a_{i}$ happens before $a_{j}$, and (2) if $a_{i}$ and $b_{j}$ are the sending and receiving of a message respectively, then $a_{i}$ happens before $b_{j}$. The happens before relation is a partial order that formalizes our intuitive notion of which events can causally affect each other. An event $a_{i}$ can only be caused by an event $b_{j}$ that happens before it. The set $\left\{b_{j} \mid b_{j} \rightarrow a_{i}\right\}$ is called the causal history of $a_{i}$.

\subsection{Data Provenance}

Given a relational database instance $I$, a relational algebra query $Q$, and a tuple $t$ in the output of the query, it is natural to ask why $t$ appears in the output. For example, consider the relational database instance given in Figure 1 that describes users and friends in a social media application. And, consider the relational algebra query

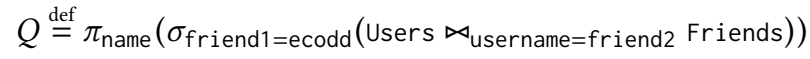

that returns the name of all of Edgar Codd's friends. Evaluating $Q$ on $I$ produces the tuple $t=$ (Michael Jordan).

Users
\begin{tabular}{|l|l|}
\hline username & name \\
\hline ecodd & Edgar Codd \\
jumpman & Michael Jordan \\
mlpro & Michael Jordan \\
\hline
\end{tabular}

\begin{tabular}{|c|c|}
\hline Friends & \\
\hline friend1 & friend2 \\
\hline $\begin{array}{l}\text { ecodd } \\
\text { ecodd }\end{array}$ & $\begin{array}{l}\text { jumpman } \\
\text { mlpro }\end{array}$ \\
\hline
\end{tabular}

Figure 1: An example database instance

Intuitively, $t=$ (Michael Jordan) is present in the output $Q(I)$ for two reasons: (1) the existence of the (ecodd, jumpman) and (jumpman, Michael Jordan) tuples and (2) the existence of the (ecodd, mlpro) and (mlpro, Michael Jordan) tuples. Why-provenance [5, 7] formalizes this intuition. The why-provenance ${ }^{1}$ of a tuple $t$ with respect to query $Q$ and database instance $I$, denoted $\operatorname{Why}(Q, I, t)$, is a set $J_{1}, \ldots, J_{n}$ of subinstances of $I$ where each subinstance $J_{i} \subseteq I$ suffices to produce $t$ (i.e. $t \in Q\left(J_{i}\right)$ ). These subinstances are called witnesses of $t$, and a witness $J_{i}$ is called a minimal witness of $t$ if no proper subinstance of $J_{i}$ is also a witness of $t$. The minimal why-provenance of $t$, denoted MWhy $(Q, I, t)$, is the set of the minimal witnesses in $\operatorname{Why}(Q, I, t)$. It can be shown that MWhy $(Q, I, t)$ is exactly the set of minimal witnesses of $t$ [7].

\footnotetext{
${ }^{1}$ For a formal definition of why-provenance, we refer the reader to [7]. For our purposes, an informal understanding of why-provenance is sufficent.
}

SoCC '18, October 11-13, 2018, Carlsbad, CA, USA

Returning to our example above, the why-provenance of the (Michael Jordan) tuple is the set $\left\{J_{1}, J_{2}\right\}$ where

$$
\begin{aligned}
& J_{1}=\{(\text { ecodd }, \text { jumpman }),(\text { jumpman, Michael Jordan })\} \\
& \left.J_{2}=\{\text { (ecodd, mlpro),(mlpro, Michael Jordan })\right\}
\end{aligned}
$$

$J_{1}$ and $J_{2}$ are minimal witnesses, so the why-provenance and minimal why-provenance of $t=$ (Michael Jordan) are the same.

\subsection{State Machines}

It is common to model servers-like key-value stores or relational databases-as deterministic state machines that repeatedly receive requests, update their state, and send replies [21, 31]. More precisely, a deterministic state machine $M=\left(S, s_{0}, \Sigma, \Lambda, \delta, \epsilon\right)$ consists of a (potentially infinite) set $S$ of states, a start state $s_{0} \in S$, an input alphabet $\Sigma$, an output alphabet $\Lambda$, a transition function $\delta: S \times \Sigma \rightarrow S$, and an output function $\epsilon: S \times \Sigma \rightarrow \Lambda$. A state machine $M$ begins in state $s_{0}$ and repeatedly receives inputs $a \in \Sigma$. Upon receiving an input $a, M$ transitions from state $s$ to state $\delta(s, a)$ and outputs $\epsilon(s, a)$.

In our work, we need to reason about specific sub-inputs to a state machine, in the spirit of why-provenance, so we introduce some notation here. We refer to an ordered sequence of inputs received by a state machine as a trace $T=a_{1} a_{2} \ldots a_{n} \in \Sigma^{*}$. A subtrace $T^{\prime}$ of $T$ is a subsequence $T^{\prime}=a_{i_{1}} a_{i_{2}} \ldots a_{i_{m}}$ where $i_{1}, i_{2}, \ldots, i_{m}$ are distinct elements of $1,2, \ldots, n$ in ascending order. Note that a subsequence does not have to be contiguous. For example, $T^{\prime}=a_{1} a_{3}$ is a subtrace of $T=a_{1} a_{2} a_{3} a_{4}$. If $T^{\prime}$ is a subtrace of $T$, then a supertrace of $T^{\prime}$ in $T$ is a subtrace of $T$ that contains every element of $T^{\prime}$. If $T_{1}$ and $T_{2}$ are two traces, we write the concatenation of $T_{1}$ and $T_{2}$ as $T_{1} T_{2}$. Similarly, if $T \in \Sigma^{*}$ is a trace, and $a \in \sum$ is an input, we let $T a$ denote the trace produced by appending $a$ to the end of $T$. An illustrative example of the definition of subtraces and supertraces is given in Figure 2.

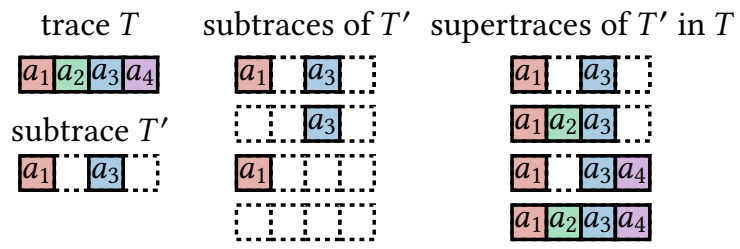

Figure 2: Subtraces and supertraces

$\delta$ takes in a state $s \in \Sigma$ and a single input $a \in \Sigma$. It is convenient to extend $\delta$ to a function $\delta^{*}: S \times \Sigma^{*} \rightarrow S$ that takes in a state $s$ and a trace $T \in \Sigma^{*}$ and outputs the state reached after sequentially executing the inputs in $T$ starting in state $s$. Similarly, we can extend $\epsilon$ to a function $\epsilon: S \times$ $\Sigma^{+} \rightarrow \Lambda$ which takes in a state $s$ and a non-empty trace 
$T=a_{1} \ldots a_{n} \in \Sigma^{+}$and returns $\epsilon\left(\delta^{*}\left(s, a_{1} a_{2} \ldots a_{n-1}\right), a_{n}\right):$ the final output produced from sequentially executing every input in $T$ starting in state $s$.

\section{WAT-PROVENANCE}

\subsection{A Motivating Example}

It is difficult to reason precisely about the causes of events in a heterogeneous distributed system. To best understand why, let's look at a simple example. Consider the implementation of a Facebook-like social media application called ZardozBook, illustrated in Figure 3. ZardozBook users post status updates, and these updates are only viewable by their friends on the site. Users send requests to a load balancer that forwards the requests to one of three weakly consistent Redis-backed application servers: $s_{1}, s_{2}$, and $s_{3}$. These application servers store a cache of ZardozBook's data in Redis and periodically synchronize their caches with a centralized Postgres database.

Consider a scenario in which Ava, a ZardozBook user, has a falling out with Bob, a friend of hers on the site. Ava unfriends Bob and then posts the status "Bob is a big jerk!", thinking that Bob will not see the status because he is no longer her ZardozBook friend. Unfortunately, Bob later logs in to ZardozBook and sees Ava's mean comment!

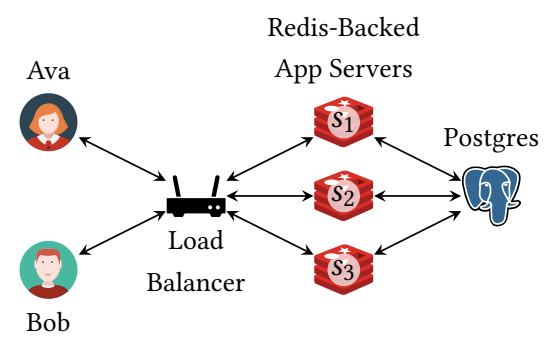

Figure 3: Social media application

Why did this happen? Here's an informal account. Ava's request to unfriend Bob was forwarded to application server $s_{1}$ by the load balancer. Then, Ava's request to post the mean comment about Bob was forwarded to $s_{2} . s_{2}$ then pushed the comment to the Postgres repository. $s_{3}$ then issued a SQL query to the Postgres repository, pulling the latest data into its Redis cache. In doing so, it pulled in Ava's mean comment. Finally, when Bob logged in, his request was forwarded to $s_{3}$ which returned the mean comment.

We argue that existing formalisms are inadequate for discovering this sequence of events. One possibility is to use causality, as described in Section 2. We could instrument our distributed system to record the causal history of every event that takes place in the system. Then, we could examine the causal history of Bob's request in an attempt to diagnose why
Bob was seeing Ava's mean comment. Unfortunately, this is not helpful. The causal history of Bob's request includes $e v$ ery event that causally precedes it, whether or not the event is relevant. For example, the causal history of Bob's request would include every message received by server $s_{3}$ prior to Bob sending his request, even those that do not involve Ava and Bob. The problem is that causality is too coarse-grained. It fails to incorporate any notion of a system's semantics as a means to filter out irrelevant messages. Instead, it returns an overapproximation of all the events that might cause an event instead of the events that do cause an event.

This might prompt us to try and apply ideas from data provenance. Unlike causality, why-provenance does incorporate system semantics to return the causes of a particular output of a query. As discussed above, why-provenance has two flaws. First is why-provenance's restriction to relational queries: while we might be able to use why-provenance to debug $s_{3}$ 's SQL query that was sent to the Postgres database, that is only one small piece of the puzzle. Understanding why Bob saw Ava's mean comment requires us to reason about messages that travel through our application servers, our Redis servers, and our load balancer. But, these are not relational databases, so we cannot apply why-provenance to them. Second and more fundamental is why-provenance's inability to reflect any notion of state change over time. In particular, the real "why" question we want to answer here is "why was the provenance of Bob's query unaffected by Ava's unfriend request?" The why-provenance of Bob's query has no answer to this question; it knows nothing about updates or the order in which they happen.

\subsection{Defining Wat-Provenance}

In isolation, causality and data provenance are both insufficient to diagnose why Bob saw Ava's mean comment. Understanding the root cause of this anomalous behavior requires us to reason about the ordering of events within the network (as with causality) and the precise data dependencies between different requests (as with data provenance). Wat-provenance unifies the benefits of causality and data provenance. Borrowing from causality, wat-provenance is a general-purpose mechanism that can be applied to arbitrary state machines. Borrowing from why-provenance, watprovenance incorporates system semantics to produce the causes of an event, rather than a conservative overapproximation.

More formally, we consider a deterministic state machine $M=\left(S, s_{0}, \Sigma, \Lambda, \delta, \epsilon\right)$, a trace $T \in \Sigma^{*}$, and a particular input $i \in \Sigma$. The state machine begins in state $s_{0}$ and executes $T$, transitioning to state $s_{T}=\delta^{*}\left(s_{0}, T\right)$. It then executes input $i$ producing output $o=\epsilon^{*}\left(s_{0}, T i\right)=\epsilon\left(s_{T}, i\right)$. Wat-provenance 
Debugging Distributed Systems with Why-Across-Time Provenance

aims to formalize an intuitive notion of why the state machine $M$ produces output $o$ when given input $i$. We build this intuitive notion by way of a sequence of examples, ultimately leading to the definition of wat-provenance.

Example 3.1. Consider a key-value server state machine $M$ with an input alphabet that consists of sets and gets to integer-valued variables that are initially 0 . Consider the trace

$$
T=\operatorname{set}(x, 1) ; \operatorname{set}(y, 2)
$$

that consists of two requests: one that sets the value of $x$ to 1 and one that sets the value of $y$ to 2 . Consider request $i=\operatorname{get}(x)$ that requests the value of $x$. When $M$ processes trace $T$ and then input $i$, it expectedly outputs 1 (i.e. $o=$ $\left.\epsilon^{*}\left(s_{0}, T i\right)=1\right)$.

Why $\operatorname{did} M$ output 1 ? In this trivial example, $M$ returned 1 as the value of $x$ because the first request in $T$ set $x$ 's value to 1. More formally, the subtrace $T^{\prime}=\operatorname{set}(x, 1)$ of $T$ suffices to generate the output of 1 (i.e. $\epsilon^{*}\left(s_{0}, T^{\prime} i\right)=o$ ). The lesson here is that the cause of an output $o$ is a subtrace of the input that is sufficient to generate $o$. We call such a subtrace a witness of $o$.

However, the entire trace $T$ is also a witness. That is, $T$ also suffices to generate an output of 1 . But, the $\operatorname{set}(y, 2)$ request is not relevant to our get $(x)$ request, so we shouldn't include it as a cause of our output. Thus, we revise our earlier observation; the cause of an output $o$ is a subtrace of the input that is sufficient to generate $o$ and is also in some sense minimal. We will define minimality more carefully below.

Example 3.2. Consider a state machine $M$ that stores a set of boolean-valued variables that are initially false. Users can set variables to true or false and can request that $M$ evaluate a formula over these variables. For example, consider the trace

$$
T=\operatorname{set}(a) ; \operatorname{set}(b) ; \operatorname{set}(c) ; \operatorname{set}(d)
$$

that sets variables $a, b, c, d$ to true. Further, consider the input $i=\operatorname{eval}((a \wedge d) \vee(b \wedge c))$ that requests $M$ evaluate the formula $(a \wedge d) \vee(b \wedge c) . o=\epsilon^{*}\left(s_{0}, T i\right)$ is expectedly true; $a, b, c, d$ are all true, so the formula evaluates to true.

Why $\operatorname{did} M$ output true? Well, there are two reasons. The first is the subtrace $T_{a d}=\operatorname{set}(a)$; $\operatorname{set}(d)$, and the second is the subtrace $T_{b c}=\operatorname{set}(b)$; $\operatorname{set}(c)$. Both of these subtraces are witnesses of $o$, so we should include both in an explanation of our output. We again revise the lesson from our previous example; the cause of an output $o$ is a set of witnesses of $o$.

Example 3.3. Consider again the state machine $M$ from the previous example, and consider the trace

$$
T=\operatorname{set}(a) ; \operatorname{set}(b) ; \operatorname{set}(c)
$$

SoCC '18, October 11-13, 2018, Carlsbad, CA, USA

and request $i=\operatorname{eval}((a \wedge \neg b) \vee c) . o=\epsilon^{*}\left(s_{0}, T i\right)$ is true. $a \wedge \neg b$ evaluates to false because $\neg b$ is false, but $c$ is true, so $(a \wedge \neg b) \vee c$ is true. Why did $M$ output true? Well, as we just explained $(a \wedge \neg b) \vee c$ is true solely because $c$ is true. Thus, the subtrace $T_{c}=\operatorname{set}(c)$ should be the only explanation. However, the subtrace $T_{a}=\operatorname{set}(a)$ is also a witness! If $M$ executes $T_{a}$ and then $i, M$ will output true.

This is certainly not what we want. set( $a$ ) does not contribute to our output, so it should be excluded. The problem here is that the subtrace $T_{a}$ does not include the set $(b)$ request that ultimately keeps the $\operatorname{set}(a)$ request from satisfying the formula. From this, we see that in order for a witness $T^{\prime}$ to be a good explanation of a particular output $o$, it must be that every supertrace of $T^{\prime}$ in $T$ is also a witness of o. $T_{a b}=\operatorname{set}(a) ; \operatorname{set}(b)$ is a supertrace of $T_{a}$ in $T$, but $T_{a b}$ does not suffice to generate $o$. Thus, $a$ is not a valid witness.

Combining our lessons from Example 3.1, Example 3.2, and Example 3.3, we arrive at our definition of wat-provenance. Given a state machine $M$, an input trace $T$, an input $i$, and the corresponding output $o=\epsilon^{*}\left(s_{0}, T i\right)$, we say that a subtrace $T^{\prime}$ of $T$ is a witness of $o$ if $\epsilon^{*}\left(s_{0}, T^{\prime} i\right)=o$. We say that a witness $T^{\prime}$ of $o$ is closed under supertrace in $T$ if every supertrace of $T^{\prime}$ in $T$ is also a witness of $o$. Let $\operatorname{Wit}(M, T, i)$ be the set of witnesses of $o$ that are closed under supertrace in $T$. The watprovenance of input $i$ with respect to $M$ and $T$, abbreviated $\operatorname{Wat}(M, T, i)$, is the set of minimal elements of $\operatorname{Wit}(M, T, i)$. That is, Wat $(M, T, i)$ consists of every witness $T^{\prime}$ of $o$ such that (1) $T^{\prime}$ is closed under supertrace in $T$, and (2) no proper subtrace of $T^{\prime}$ is also a witness of $o$ that satisfies $(1)^{2}$. Note that we formally define wat-provenance with respect to an input $i$, but colloquially discuss wat-provenance with respect to the corresponding output $o$.

\subsection{Can I Get a Witness?}

We now provide a few more simple examples involving watprovenance to illustrate the definition. In Section 4, we describe the wat-provenance of realistic services such as Redis, Zookeeper, and S3.

Example 3.4. Consider again the key-value server state machine from Example 3.1, the trace

$$
T=a_{1} a_{2} a_{3}=\operatorname{set}(x, 1) ; \operatorname{set}(x, 2) ; \operatorname{set}(x, 1)
$$

and the input $i=\operatorname{get}(x) . o=\epsilon^{*}\left(s_{0}, T i\right)=1$. To compute Wat $(M, T, i)$ (the wat-provenance of $o$ ), we first compute

\footnotetext{
${ }^{2}$ Note the subtlety that to find $\operatorname{Wat}(M, T, i)$, we first list all the witnesses of $o$ that are closed under supertrace and then remove the non-minimal elements. We do not list all the minimal witnesses and then remove the ones that are not closed under supertrace in $T$. Informally, wat-provenance is minimal(closed_under_supertrace(witnesses)), not closed_under_supertrace(minimal(witnesses)). These two are not the same. See Example 3.5, for example.
} 
Wit $(M, T, i)$ : the witnesses of $o$ that are closed under supertrace in $T$.

$T_{3}=a_{3}=\operatorname{set}(x, 1)$ suffices to generate $o$ and is closed under supertrace in $T$ because the proper supertraces $a_{1} a_{3}$, $a_{2} a_{3}$, and $a_{1} a_{2} a_{3}$ all generate $o$. So, $T_{3} \in \mathrm{Wit}(M, T, i)$. By a similar line of reasoning, we also find that $a_{1} a_{3}, a_{2} a_{3}$, and $a_{1} a_{2} a_{3}$ are in $\operatorname{Wit}(M, T, i)$.

The subtrace $T_{1}=a_{1}=\operatorname{set}(x, 1)$ is also a witness of $o$, but it is not closed under supertrace in $T$ because the supertrace $a_{1} a_{2}=\operatorname{set}(x, 1) ; \operatorname{set}(x, 2)$ does not generate $o$. Thus, $T_{1}$ is not in $\operatorname{Wit}(M, T, i)$ and therefore not in $\operatorname{Wat}(M, T, i)$. Intuitively this is correct because it is the second set $(x, 1)$ command, not the first, that causes the value of $x$ to ultimately be 1 .

All other subtraces of $T$ are not witnesses of $o$. Thus, $\operatorname{Wit}(M, T, i)=\left\{a_{3}, a_{1} a_{3}, a_{2} a_{3}, a_{1} a_{2} a_{3}\right\}$ which has unique minimal element $a_{3}$. Thus, Wat $(M, T, i)=\left\{a_{3}\right\}$.

Example 3.5. Consider again the key-value server state machine from Example 3.1 and Example 3.4 with the input alphabet expanded to include additions and subtractions to a particular variable. Consider the trace

$$
T=a_{1} a_{2} a_{3} a_{4}=\operatorname{set}(x, 42) ; \operatorname{add}(x, 1) ; \operatorname{add}(x, 2) ; \operatorname{sub}(x, 3)
$$

and the request $i=\operatorname{get}(x) . o=\epsilon^{*}\left(s_{0}, T i\right)=42$.

Again, we compute $\operatorname{Wit}(M, T, i)$. $T$ suffices to generate $o$, and $T$ does not have any proper supertraces in $T$, so it is trivially closed under supertrace in $T$. Thus, $T \in \mathrm{Wit}(M, T, i)$. $T_{1}=a_{1}=\operatorname{set}(x, 42)$ is the only other witness of $o$, but $T_{1}$ is not closed under supertrace in $T . T_{12}=\operatorname{set}(x, 42)$; $\operatorname{add}(x, 1)$ is a supertrace of $T_{1}$ but does not generate $o$. Thus, $\operatorname{Wit}(M, T, i)=$ $\operatorname{Wat}(M, T, i)=\{T\}$.

Note that we first compute the witnesses that are closed under supertrace in $T$ and then remove the non-minimal elements. Imagine instead if we had first computed the minimal witnesses and then removed the elements that were not closed under supertrace in $T$. We would have found that the sole minimal witness of $o$ was $T_{1}$. Then, we would have filtered out $T_{1}$ because, as we just saw, it is not closed under supertrace in $T$. This would leave us with an empty wat-provenance!

Example 3.6. Consider a relational database state machine $M$. The input alphabet of $M$ includes commands to insert a tuple into $M$ and to execute a relational algebra query against $M$. Initially, all relations are empty. Consider the trace

$$
T=a_{1} a_{2} a_{3}=\operatorname{insert}(R, t) ; \operatorname{insert}(R, u) ; \operatorname{insert}(S, u)
$$

that inserts tuple $t$ into relation $R$, inserts tuple $u$ into relation $R$, and inserts tuple $u$ into relation $S$. Consider the request $i=$ query $(R-S)$ that queries the set difference $R-S$ of $R$ and $S . o=\epsilon^{*}\left(s_{0}, T i\right)=\{t\}$ is the set of only the tuple $t$.
We first compute $\operatorname{Wit}(M, T, i)$. There are only three witnesses of $o: a_{1}, a_{1} a_{3}$, and $a_{1} a_{2} a_{3} . a_{1}$ is not closed under supertrace in $T$ because the supertrace $a_{1} a_{2}$ does not generate $o$. The other two traces, $a_{1} a_{3}$ and $a_{1} a_{2} a_{3}$, are closed under supertrace. Thus, Wit $(M, T, i)=\left\{a_{1} a_{3}, a_{1} a_{2} a_{3}\right\}$, and $\operatorname{Wat}(M, T, i)=\left\{a_{1} a_{3}\right\}$.

\subsection{Wat-Provenance Properties}

Example 3.1 through Example 3.6 demonstrate that our definition of wat-provenance accurately models our intuition about data provenance for state machines. We now discuss how wat-provenance relates to why-provenance and causality.

\section{Claim 1. Wat-provenance subsumes why-provenance.}

Intuitively, wat-provenance generalizes why-provenance from relational databases to arbitrary state machines. We can formalize this intuition in the following way.

Let $M$ be a general relational database state machine (first introduced in Example 3.6) that allows for the insertions of tuples and the execution of monotone relational queries (i.e. queries composed of the monotone relational algebra operators select, project, join and union). Let $I$ be an arbitrary database instance, and let $T$ be a trace which inserts every tuple in $I$. Let input $i=\operatorname{in}(t, Q)$ be an input which returns a boolean that indicates whether tuple $t$ is in the result of evaluating query $Q$ on instance $I$. Then, viewing a subtrace $T^{\prime}$ as a subinstance $I^{\prime} \subseteq I$, Wat $(M, T, i)=\operatorname{MWhy}(Q, I, t)$.

Proving this fact is straightforward. If $t \notin Q(I)$, then $o=\epsilon^{*}\left(s_{0}, T i\right)$ returns false and $\operatorname{Wat}(Q, I, t)$ consists only of the empty trace, indicating that $\operatorname{MWhy}(Q, I, t)$ is empty. Otherwise $t \in Q(I)$ and $o$ returns true. Consider a witness $T^{\prime} \in \operatorname{Wat}(M, T, i)$. $T^{\prime}$ suffices to generate $o$, so the corresponding instance $I^{\prime}$ suffices to generate $t$. Moreover, because $Q$ is monotone and $T$ does not contain any deletions, every witness $T^{\prime}$ is closed under supertrace in $T$. Thus, $T^{\prime}$ (and hence $I^{\prime}$ ) is a minimal witness. The proof of the converseshowing that every $I^{\prime} \in M W h y(Q, I, t)$ has a corresponding subtrace $T^{\prime} \in$ Wat $(M, I, t)$-is symmetric.

Unfortunately, wat-provenance's generality does not come for free. Given a query $Q$, tuple $t$, and instance $I$, it is possible to automatically compute $\operatorname{MWhy}(Q, I, t)$ because queries are constructed from a fixed set of simple relational operators. As we discuss in Section 4, it is normally intractable to automatically compute the wat-provenance of a particular state machine because, unlike relational queries, these state machines can have arbitrarily complex semantics.

\section{Claim 2. Wat-provenance refines causality.}

While wat-provenance generalizes why-provenance, it refines causality in the following sense. Consider a state machine $M$ that executes a trace $T$ and then an input request $i$. 
Debugging Distributed Systems with

Why-Across-Time Provenance

The causal history of $i$ includes every single request in $T$ (and the causal history of every request in $T$ ) whether or not the request in $T$ actually did influence the output of executing request $i$. Wat-provenance instead returns the subtraces of $T$ that are actual causes.

Note that wat-provenance returns a set of witnesses that are local to a particular node. Wat-provenance does not return the causal history of these witnesses, which includes messages sent by other nodes in the distributed system. In Section 5, we see how to enrich wat-provenance with this information.

\subsection{Limitations}

To clarify the strengths of wat-provenance, we pause to discuss its limitations.

Wat-provenance is not GDB. Wat-provenance is a highlevel debugging technique. It can be used to identify the necessary and sufficient inputs that cause a state machine to produce a particular output (the why), but unlike low-level debugging tools like GDB, it cannot describe the details of the code that actually produces a particular output (the how).

To make things concrete, consider again our motivating example from Section 3.1 in which two of Ava's requests were reordered, allowing Bob to erroneously see Ava's mean comment. We can use wat-provenance to debug scenarios like this one. These types of scenarios require us to reason about which messages affect other messages, to reason about how events are ordered with respect to one another, and to reason about how data is transferred across multiple machines across a span of time. On the other hand, wat-provenance cannot help us understand why, for example, a particular node is segfaulting; we need a lower-level tool like GDB for this.

Thus, we view wat-provenance and GDB-like debuggers as complementary. When debugging, a developer can use wat-provenance to trace the root cause of a bug across a system, narrowing their attention down to only the relevant inputs. Then, if needed, they can use a low-level debugger like GDB to discover the details of what's going wrong.

Wat-provenance requires determinism. Wat-provenance is defined with respect to deterministic state machines, yet many pieces of code are nondeterministic. For example, the behavior of many weakly consistent distributed systems depend on the non-deterministic ordering of messages in the network. Similarly, some load balancers intentionally use randomness when deciding which machine should receive a particular request. Wat-provenance specifications cannot be applied to nondeterministic systems like these. We leave a generalization of wat-provenance to nondeterministic state machines as an interesting avenue for future work.
SoCC '18, October 11-13, 2018, Carlsbad, CA, USA

\section{WAT-PROVENANCE SPECIFICATIONS}

Now that we have defined wat-provenance, we turn to the matter of computing it.

\subsection{Provenance Specifications}

Automatically computing the wat-provenance for an arbitrary distributed system component, which we dub a black box, is often intractable and sometimes impossible. Computing the wat-provenance of a black box requires that we analyze the black box's implementation to extract the relationships between the inputs and outputs of the black box. Because black box implementations can be large and complex, this program analysis is almost always intractable. Worse, we may not have access to the source code of the black box at all. For example, cloud services like Amazon S3 or Google Cloud Spanner have proprietary implementations. In this case, automatically computing wat-provenance is impossible.

Though automatically computing the wat-provenance for an arbitrary black box is intractable, we can take advantage of the fact that many real-world black boxes are far from arbitrary. Many black boxes have complex implementations but are designed with very simple interfaces. This allows us to sidestep the issue of inferring wat-provenance from an implementation and instead specify wat-provenance directly from an interface. That is, we can write a wat-provenance specification: a function that-given a trace $T$ and request $i$-directly returns the wat-provenance $\operatorname{Wat}(M, T, i)$ for a black box modeled as state machine $M$.

For example, if we restrict our attention to the get and set API of Redis, then the wat-provenance specification of a get request is trivial: the wat-provenance of a get request for key $k$ includes only the most recent set to $k$. Redis is implemented in over 50,000 lines of C. Analyzing this body of code and inferring the wat-provenance of a get request is infeasible using modern program analysis techniques. Watprovenance specification avoids this issue entirely and instead specifies the wat-provenance in a single line of text.

Moreover, codifying this one-line wat-provenance specification is expectedly straightforward. Wat-provenance specifications are implemented as functions that take in a trace $T$ and an input $i$ and return the wat-provenance of $i$. Thus, watprovenance specifications can be written in any programming language and can use any language features available. Wat-provenance specifications do not have to be written using any special domain specific language or using any restricted subset of a language.

As a simple example, we provide a Python implementation of the wat-provenance specification in Figure 4. The specification, get_prov, takes in a trace $T$ and a get request $i$ for key $k$. Redis requests are represented as objects of type Request with subclasses GetRequest and SetRequest. 
get_prov iterates through the trace in reverse order, looking for a set request to key $k$. If such a set request is found, get_prov returns it. Otherwise, get_prov returns an empty witness.

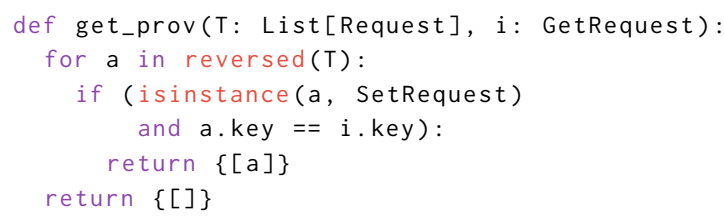

Figure 4: A Python implementation of a Redis get request wat-provenance specification

In Section 5, we present a prototype implementation of a system for writing wat-provenance specifications and describe the details of how the system collects traces. In this section, we omit these details and focus on the concepts behind wat-provenance specifications.

\subsection{Examples}

The wat-provenance specification of a Redis get request is particularly simple, but in our experience this simplicity is not completely uncommon. We now survey a variety of commonly used black boxes that have relatively simple wat-provenance specifications. Later, we discuss some black boxes for which writing wat-provenance specifications is more difficult.

Key-Value Stores. We have already seen a wat-provenance specification for the get and set API of Redis. We can easily extend our wat-provenance specification to handle more of Redis' API. For example, consider the operations append, decr, decrby, incr, and incrby that all modify the value associated with a particular key. With these operations present in a trace, the wat-provenance specification for a get request to key $k$ now includes the most recent set to $k$ and all subsequent modifying operations to key $k$.

Object Stores. We can also write wat-provenance specifications for storage systems that are more complex than key-value stores. For example, consider an object store like Amazon S3 where users can create, move, copy, list, and get buckets and objects. The wat-provenance specification for the get of an object $o$ in bucket $b$ includes the most recent creation of the bucket $b$ and the most recent creation of $o$. If a bucket or object was created by a move, then the provenance also includes the provenance of the moved bucket or object. The wat-provenance of a request to list the contents of a bucket includes the most recent creation of the bucket, the most recent creation of every object in the bucket, and the deletion of any object that was previously in the bucket.
Distributed File Systems. We can specify the wat-provenance of a distributed file system like HDFS. A wat-provenance specification of a request to read a byte range from a file includes the most recent creation of the file, the most recent creation of the parent directories of the file, and the most recent writes that overlap with the requested byte range. If the file was created by moving another file, then the watprovenance specification also includes the provenance of the file that was moved.

Coordination Services. Systems use coordination services like Apache Zookeeper [16] and Chubby [6] for leader election, mutual exclusion, etc. Take Zookeeper as an example. Zookeeper's API resembles that of a file system; users can create, delete, write, and read file-like objects called znodes. Though the implementation of Zookeeper and HDFS are radically different, their APIs (and thus their wat-provenance specifications) are similar. For example, the wat-provenance specification of a request to read a znode includes the most recent creation of the znode and the most recent creations of all ancestor znodes.

Load Balancers. Consider a load balancer, like HAProxy, that is balancing load across a set $s_{1}, \ldots, s_{n}$ of $n$ servers. Periodically, a server $s_{i}$ sends a heartbeat to the load balancer that includes $s_{i}$ 's average load for the last five minutes. Whenever the load balancer receives a message from a client, it forwards the message to the server $s_{i}$ that is least loaded. Modelling the forwarding decision $s_{i}$ as the output of the load balancer, the wat-provenance specification for the forwarding decision includes the most recent heartbeat message from the least-loaded server.

Stateless Services. A stateless service is a service that can be modelled as a state machine with a single state. Given a request, a stateless service always produces the same reply, no matter what other requests it has already serviced. For example, a web server serving a static website is stateless; it replies to all requests with the same website. Wat-provenance specifications of a stateless service are trivial. Requests are completely independent, so the wat-provenance of any request consists only of the empty witness.

\subsection{Limitations}

Though wat-provenance specifications are often simple to write, they are not a panacea. Here, we discuss some limitations of wat-provenance specifications.

Complex wat-provenance specifications. There are some black boxes for which writing a wat-provenance specification is inherently very difficult. For example, consider a state machine that implements an online support vector machine (SVM) $[4,15,22]$. Clients can either (a) submit training data to 
Debugging Distributed Systems with

Why-Across-Time Provenance

the state machine to update the model or (b) submit test data to the state machine for classification. The wat-provenance of a classification request includes only the support vectors of the model at the time of classification. Writing a watprovenance specification to identify these support vectors is possible but very difficult. Such a wat-provenance specification would likely be as complicated as the state machine itself.

Buggy black boxes. We have thus far tacitly assumed that black boxes like Redis faithfully implement their advertised interfaces. However, if a black box is buggy and deviates from its expected behavior, then a wat-provenance specification will produce erroneous provenance. In other words, watprovenance specifications are written with respect to the semantics of an abstract state machine. If a particular black box does not concretely implement these semantics, then the wat-provenance specification is incorrect.

\section{WATERMELON}

In this section, we present Watermelon: a prototype distributed debugging framework that leverages the theoretical foundations of wat-provenance and wat-provenance specifications.

Watermelon uses wat-provenance specifications to generate the provenance of data as it transits through the black box components of a distributed system. To write a watprovenance specification for a black box, a developer must first wrap the black box in a Watermelon shim. A shim acts as proxy, intercepting all inbound requests sent to a black box and all outbound replies produced by a black box. Watermelon shims provide two key pieces of functionality.

First, Watermelon shims are responsible for recording the trace $T$ of requests that are sent to a black box, as well as the corresponding replies produced by the black box. These traces are later used as the inputs to wat-provenance specifications. Currently, Watermelon shims persist traces in a relational database.

Second, Watermelon shims implement a simple distributed tracing service. Whenever a Watermelon shim receives a request, it records the address of the message's sender along with the request. Similarly, whenever a Watermelon shim sends a request, it records the address of the message's destination. This enables a developer to integrate the watprovenance of multiple black boxes within a distributed system. To find the cause of a particular black box output, we invoke the black box's wat-provenance specification. The specification returns the set of witnesses that cause the output. Then, we can trace a request in a witness back to the black box that sent it and repeat the process, invoking the sender's wat-provenance specification to get a new set of witnesses.

\section{SoCC '18, October 11-13, 2018, Carlsbad, CA, USA}

After a user has written a black box's shim, they can write the black box's wat-provenance specification. Watermelon wat-provenance specifications are simple scripts written in a developer's choice of either SQL or Python. Given a particular black box request, a wat-provenance script computes the corresponding wat-provenance with respect to the black box's trace (which is persisted in a relational database by the black box's shim).

\section{EVALUATION}

In this section, we answer two questions: (1) How difficult is it to write wat-provenance specifications? and (2) How do wat-provenance and wat-provenance specifications compare to other debugging techniques? We answer question 1 in Section 6.1 and question 2 in Section 6.2.

\subsection{Wat-Provenance Specifications}

In Section 4, we argued that many commonly used distributed system components have relatively simple wat-provenance specifications. In this subsection, we substantiate our argument with concrete wat-provenance specifications for Redis, a subset of the POSIX file system API, Amazon S3, and Zookeeper. We implemented these wat-provenance specifications using Watermelon. Table 1 lists the language in which we wrote each provenance specification, the lines of code required to write each specification, the number of APIs supported by each specification, and the API supported by each specification.

We found it simple to write wat-provenance specifications for 17 of the 20 APIs. We found specifying three of the APIs slightly more challenging, but still relatively straightforward. First, specifying the read of a byte range in a file system required us to find the most recent write to each segment of the byte range. This required us to scan backwards through the trace, maintaining a disjoint set of byte ranges. Second, specifying the catting and listing of objects in Amazon S3 was complicated by the fact that objects could be moved across buckets. Thus, computing the wat-provenance required computing the transitive wat-provenance of objects that have been moved and copied.

As we discussed in Section 4.3, this does not mean that all black boxes have simple wat-provenance specifications, but it corroborates the claim that many commonly used black boxes do.

\subsection{Debugging with Wat-Provenance}

How do wat-provenance and wat-provenance specifications compare to other debugging techniques? We answer this question by comparing Watermelon, our prototype watprovenance debugger, against two other debugging techniques: SPADE [12] and printf debugging. We qualitatively 
Table 1: Watermelon wat-provenance specifications

\begin{tabular}{lllll}
\hline System & Language & LOC & Number of APIs & Supported API \\
\hline Redis & SQL & 30 & 9 & get, set, del, append, incr, decr, incrby, decrby, strlen \\
POSIX & Python & 88 & 2 & reading and writing byte ranges \\
Amazon S3 & Python & 200 & 5 & creating, copying, catting, removing, and listing objects and buckets \\
Zookeeper & SQL & 70 & 4 & creating, reading, writing, and listing znodes \\
\hline
\end{tabular}

evaluate the three debugging techniques using four metricsease of adoption, runtime overhead, high-level debugging support, and low-level debugging support-which we will explain momentarily. This qualitative analysis is summarized in Table 2.

6.2.1 SPADE. SPADE [12] is a framework that collects provenance information from arbitrary black boxes in a distributed system. SPADE collects provenance information from a variety of sources including operating system audit logs, network artifacts, LLVM instrumented applications, and applications dynamically instrumented for taint analysis. These techniques are the current state of the art in extracting the provenance of unmodified black boxes. For the sake of brevity, we will focus only on the provenance that SPADE collects from operating system audit logs and LLVM instrumented applications.

Ease of Adoption. Our first metric, ease of adoption, is a measure of how difficult it is for a developer to set up the infrastructure required to use a particular debugging technique. Ease-of-adoption does not include the difficulty of actually debugging using a debugging technique; it only includes the difficulty of making a system amenable to the debugging technique. The ease of adoption of SPADE, for example, is very low. For SPADE to collect operating system audit logs, binaries can be run unmodified. To collect LLVM call graphs, binaries have to be compiled with LLVM instrumentation, but the code itself remains unchanged.

Runtime Overhead. Our second metric, runtime overhead, is a measure of the performance overheads that a debugging technique imposes on a system. When SPADE collects operating system audit logs, it imposes a negligible amount of runtime overhead, as operating system audit logs are created whether or not SPADE is being used. LLVM instrumented binaries, on the other hand, run significantly slower than their uninstrumented counterparts. As a simple example, on an Amazon EC2 m5.xlarge instance, we measured that a single Redis client required 2.45 seconds to send 100,000 synchronous PING operations to a default configured Redis server running on the same machine. When we compiled the Redis server with LLVM instrumentation, the client required 29.76 seconds, an order of magnitude decrease in throughput.
High- and Low-Level Debugging Support. Our third and fourth metrics, high-level debugging support and lowlevel debugging support, are measures of whether a debugging technique facilitates high-level and low-level debugging. As we described in Section 3.5, high-level debugging involves understanding which events in a distributed system cause each other, how events are ordered with respect to one another, etc. Conversely, low-level debugging involves reasoning about the details of how a particular program executes.

We ran SPADE-with audit logging and LLVM instrumentation enabled-against a trivial workload consisting of a single set and get to Redis. SPADE produced 1,087 audit log provenance entries and 1,118,764 LLVM instrumentation provenance entries. High-level debugging with either of these sources of provenance is difficult due to the provenance's size and detailed nature. Understanding the reported provenance requires either an understanding of how particular syscalls relate to Redis' source code (for audit logs) or a detailed understanding of Redis' implementation (for LLVM instrumentation). Low-level debugging with audit logs is challenging, because the audit logs lack information about the execution of the code being debugged (besides the syscalls that it makes). Low-level debugging with LLVM instrumentation is easier but still challenging due to the sheer volume of provenance information produced.

6.2.2 printf Debugging. "printf debugging" is the adhoc debugging technique in which a developer adds printf statements (or log statements) to the various components of a distributed system and then debugs the system by analyzing the resulting logs.

Ease of Adoption. The ease of implementing printf debugging depends on (a) how many log statements a developer wants to add and (b) the piece of code to which a developer wants to add log statements. It is relatively easy for a developer to add logging to a piece of code that they wrote because they know where and what to log. If the code is a complex piece of open source software (e.g. Apache Cassandra, Apache Zookeeper), then adding log statements requires understanding at least part of the software's implementation. 
Debugging Distributed Systems with

Why-Across-Time Provenance

SoCC '18, October 11-13, 2018, Carlsbad, CA, USA

Table 2: A qualitative comparison of debugging techniques

\begin{tabular}{lllll}
\hline Debugging Technique & Ease Of Adoption & Runtime Overheads & $\begin{array}{l}\text { Supports High-Level } \\
\text { Debugging }\end{array}$ & $\begin{array}{l}\text { Supports Low-Level } \\
\text { Debugging }\end{array}$ \\
\hline SPADE (Audit Logs) & easy & low & no & some \\
SPADE (LLVM) & easy & high & no & yes \\
printf Debugging & easy-impossible & low & some & yes \\
Watermelon & easy-hard & medium & yes & no \\
\hline
\end{tabular}

If the code is closed-source (e.g. Cloud Spanner, Amazon Redshift), then adding log statements is impossible.

Runtime Overheads. The overheads of logging are typically negligible compared to heavier weight techniques like LLVM instrumentation or Watermelon shims.

High- and Low-Level Debugging Support. The ability to perform high- and low-level debugging with log statements is challenging to characterize. It varies from easy to difficult based on the complexity of the bug being debugged, the quantity and quality of logs, etc. Still, for high-level debugging, there is an unavoidable burden imposed by printf debugging. Developers have to make sure enough information is logged, collect logs from a number of machines (some of which might have crashed), filter out irrelevant entries from the potentially voluminous logs, and trace data across multiple logs.

\subsubsection{Watermelon.}

Ease of Adoption. To adopt Watermelon, a developer must first write wat-provenance specifications for each component in the system. As we discussed, many distributed system components have relatively simple wat-provenance specifications for which adopting Watermelon is relatively easy, while some components have very complex wat-provenance specifications that make Watermelon more challenging to adopt.

Runtime Overheads. Watermelon shims introduce a nonnegligible amount of runtime overhead by intercepting network messages and periodically persisting traces. We measured the performance overheads of Watermelon shims on trivial workload in which a Redis client performs a series of SET operations against a Redis server. Watermelon shims decreased the system's throughput by $41.4 \%$. While some of this performance degradation is an artifact of our prototype, some is inherent to shims.

High- and Low-Level Debugging Support. Watermelon's greatest strength is that it makes high-level debugging easy. Wat-provenance formalizes the intuitive notion of which inputs in a distributed system cause a particular output. Watprovenance specifications allow a developer to codify these intuitions and automatically use them to filter irrelevant information when debugging. By refining causality, watprovenance also allows developers to reason about the ordering of events in a distributed system without having to manually trace events through a set of logs. Conversely, Watermelon must be paired with another system to support low-level debugging.

As a simple experiment, we again ran our trivial workload consisting of a single set and get to Redis. Whereas SPADE produced 1,089 audit $\log$ provenance entries and 1,118,764 LLVM instrumentation provenance entries, Watermelon produced 8 provenance entries: the get and set request and response recorded on the client and the server.

\section{RELATED WORK}

Data Provenance. When discussing data provenance, we have focused primarily on why-provenance, as wat-provenance is a generalization of why-provenance. However, there are other forms of data provenance. How-provenance [13] formalizes not only why a particular output is produced by a relational query, but also how. Where-provenance [5] formalizes the set of input attributes that contribute to an output attribute. In [8], Cui et al. provide algorithms for computing the provenance of more generic data transformation operators that satisfy certain properties (e.g., homomorphisms, aggregators). In [36], Woodruff et al. use weak inverse functions to compute the provenance of generic operators. Why-, how-, and where-provenance are all limited to the domain of relational queries, and all five forms of provenance are limited to the domain of data transformations applied to static data. Wat-provenance is applicable to state machines with state that varies over time.

Black Box Provenance. RDataTracker [23], noWorkflow [27], and SPADE [12] are frameworks that attempt to record the provenance of data through an arbitrary black box using general purpose low-level provenance tracing techniques. RDataTracker and noWorkflow use reflection and runtime information to track the provenance of data through minimally annotated $\mathrm{R}$ and Python scripts. SPADE collects provenance 
information from operating system audit logs, network artifacts, LLVM instrumented applications, and applications dynamically instrumented for taint analysis. The benefit of these frameworks is their generality. However, their generality comes at the cost of verbosity. These provenance frameworks produce a large amount of low-level implementationspecific provenance information that can be challenging to interpret.

Data-Dependent Process Provenance. A data-dependent process (DDP) is a finite state machine that evaluates queries against a relational database to determine some transitions and uses external requests to trigger other transitions [9, 10]. In [9] and [10], Deutch et al. extend provenance semirings [13] to linear temporal logic formulas issued against a DDP. Both DDP provenance and wat-provenance aim to extend traditional data provenance to state machines, but they do so in very different ways. DDP provenance is used to explain how particular execution traces may arise. Watprovenance, on the other hand, aims to explain why a state machine generates a particular output with respect to a fixed trace of inputs.

Network Provenance. Network provenance was originally introduced in [41] as a generalization of data provenance to programs written in the extended relational language NDLog [25]. ExSPAN [41] and DistTape [40] are two accompanying implementations that record the network provenance of a distributed system implemented in NDLog. The network provenance of a piece of data is tied to the particular NDLog program that created it, whereas wat-provenance is defined with respect to abstract state machines instead of concrete implementations. As a result, network provenance provides finer grained debugging information than wat-provenance, but is applicable only to systems written in NDLog. Watprovenance provides coarser grained debugging information, but is applicable to arbitrary state machines.

Scientific Workflow Systems. Scientific workflow systems like Taverna [35], Kepler [1], Pegasus [19], and Swift [37] can be used to structure complex computational tasks as a graph, composing tasks by connecting the outputs of one task to the inputs of another. These systems are limited to the workflow languages that they support, and these languages are not typically used to build distributed systems. They also assume that the inputs to a workflow are static. Watprovenance formalizes provenance for arbitrary distributed systems composed of time-varying state machines.

DISC Provenance. Data intensive scalable computing (DISC) frameworks like Apache Hadoop [32] and Apache Spark [38] can execute data-parallel programs on massive amounts of data both efficiently and with fault tolerance. Work on GMRW provenance [17] and systems like BigDebug [14],
Titian [18], RAMP [28], and Newt [24] augment DISC frameworks with data provenance. Unlike wat-provenance, DISC provenance is restricted to the domain of data intensive computations over static data and cannot be applied to other distributed systems components like storage systems, coordination services, load balancers, etc. DISC provenance frameworks take advantage of operator interfaces (e.g., map, filter, aggregate) to construct lineage of arbitrary operator implementations similar to how wat-provenance specifications are written against high-level interfaces instead of implementations.

Distributed Tracing Tools. Distributed tracing tools like Dapper [33], X-trace [11], Magpie [3], Stardust [34], and lprof [39] allow programmers to trace messages through large and complex distributed systems that span multiple nodes and administrative domains. The fundamental distinction between distributed traces and wat-provenance is that distributed traces do not carry historical information that link prior inputs to present outputs. For example, traces may show you which clients contacted a key-value store and when, but they will not show you which requests wrote the values that later requests read back. Also note that lprof, unlike other tracing tools, constructs traces by analyzing systems' existing logs. In the future, we plan to explore whether we can use a similar technique to construct traces.

\section{CONCLUSION}

This paper identified inadequacies in existing formalisms used to reason about the causes of events in distributed systems. Causality overapproximates the true cause of an event, and data provenance is restricted to the domain of static (typically relational) data. We then presented wat-provenance: a novel form of provenance that generalizes why-provenance and refines causality. We then discussed how to sidestep the complexity of automatic wat-provenance extraction with wat-provenance specifications written against the high-level API of a black box.

\section{ACKNOWLEDGMENTS}

We thank Lennart Oldenburg, Sanjay Krishnan, Alvin Cheung, and Anthony Tan for fruitful discussion and feedback. We also thank our shepherd, Ken Yocum, for his helpful insights and guidance. This research is supported in part by DHS Award HSHQDC-16-3-00083, NSF CISE Expeditions Award CCF-1139158, National Science Foundation Grant No. 1652368, and gifts from Alibaba, Amazon Web Services, Ant Financial, CapitalOne, Ericsson, GE, Google, Huawei, Intel, IBM, Microsoft, Scotiabank, Splunk and VMware. 


\section{REFERENCES}

[1] Ilkay Altintas, Oscar Barney, and Efrat Jaeger-Frank. 2006. Provenance collection support in the kepler scientific workflow system. In International Provenance and Annotation Workshop. Springer, 118-132.

[2] Peter Bailis, Alan Fekete, Ali Ghodsi, Joseph M Hellerstein, and Ion Stoica. 2012. The potential dangers of causal consistency and an explicit solution. In Proceedings of the Third ACM Symposium on Cloud Computing. ACM.

[3] Paul Barham, Rebecca Isaacs, Richard Mortier, and Dushyanth Narayanan. 2003. Magpie: Online Modelling and Performance-aware Systems.. In HotOS. 85-90.

[4] Antoine Bordes and Léon Bottou. [n. d.]. The Huller: a simple and efficient online SVM. ([n. d.]).

[5] Peter Buneman, Sanjeev Khanna, and Tan Wang-Chiew. 2001. Why and where: A characterization of data provenance. In International conference on database theory. Springer, 316-330.

[6] Mike Burrows. 2006. The Chubby lock service for loosely-coupled distributed systems. In Proceedings of the 7th symposium on Operating systems design and implementation. USENIX Association, 335-350.

[7] James Cheney, Laura Chiticariu, Wang-Chiew Tan, et al. 2009. Provenance in databases: Why, how, and where. Foundations and Trends® in Databases 1, 4 (2009), 379-474.

[8] Yingwei Cui and Jennifer Widom. 2003. Lineage tracing for general data warehouse transformations. The VLDB fournal-The International fournal on Very Large Data Bases 12, 1 (2003), 41-58.

[9] Daniel Deutch, Yuval Moskovitch, and Val Tannen. 2014. A provenance framework for data-dependent process analysis. Proceedings of the VLDB Endowment 7, 6 (2014), 457-468.

[10] Daniel Deutch, Yuval Moskovitch, and Val Tannen. 2015. Provenancebased analysis of data-centric processes. The VLDB fournal 24, 4 (2015), 583-607.

[11] Rodrigo Fonseca, George Porter, Randy H Katz, Scott Shenker, and Ion Stoica. 2007. X-trace: A pervasive network tracing framework. In Proceedings of the 4th USENIX conference on Networked systems design \& implementation. USENIX Association.

[12] Ashish Gehani and Dawood Tariq. 2012. SPADE: support for provenance auditing in distributed environments. In Proceedings of the 13th International Middleware Conference. Springer-Verlag New York, Inc., 101-120.

[13] Todd J Green, Grigoris Karvounarakis, and Val Tannen. 2007. Provenance semirings. In Proceedings of the twenty-sixth ACM SIGMODSIGACT-SIGART symposium on Principles of database systems. ACM, 31-40.

[14] Muhammad Ali Gulzar, Matteo Interlandi, Seunghyun Yoo, Sai Deep Tetali, Tyson Condie, Todd Millstein, and Miryung Kim. 2016. Bigdebug: Debugging primitives for interactive big data processing in spark. In Proceedings of the 38th International Conference on Software Engineering. ACM, 784-795.

[15] Marti A. Hearst, Susan T Dumais, Edgar Osuna, John Platt, and Bernhard Scholkopf. 1998. Support vector machines. IEEE Intelligent Systems and their applications 13, 4 (1998), 18-28.

[16] Patrick Hunt, Mahadev Konar, Flavio Paiva Junqueira, and Benjamin Reed. 2010. ZooKeeper: Wait-free Coordination for Internet-scale Systems. In USENIX annual technical conference, Vol. 8. Boston, MA, USA.

[17] Robert Ikeda, Hyunjung Park, and Jennifer Widom. [n. d.]. Provenance for Generalized Map and Reduce Workflows. In CIDR 2011. Stanford InfoLab. http://ilpubs.stanford.edu:8090/985/

[18] Matteo Interlandi, Kshitij Shah, Sai Deep Tetali, Muhammad Ali Gulzar, Seunghyun Yoo, Miryung Kim, Todd Millstein, and Tyson Condie. 2015 Titian: Data provenance support in spark. Proceedings of the VLDB
Endowment 9, 3 (2015), 216-227.

[19] Jihie Kim, Ewa Deelman, Yolanda Gil, Gaurang Mehta, and Varun Ratnakar. 2008. Provenance trails in the wings/pegasus system. Concurrency and Computation: Practice and Experience 20, 5 (2008), 587-597.

[20] Leslie Lamport. 1978. Time, clocks, and the ordering of events in a distributed system. Commun. ACM 21, 7 (1978), 558-565.

[21] Leslie Lamport. 1998. The part-time parliament. ACM Transactions on Computer Systems (TOCS) 16, 2 (1998), 133-169.

[22] Pavel Laskov, Christian Gehl, Stefan Krüger, and Klaus-Robert Müller. 2006. Incremental support vector learning: Analysis, implementation and applications. fournal of machine learning research 7, Sep (2006), 1909-1936.

[23] Barbara S Lerner and Emery R Boose. 2014. Collecting provenance in an interactive scripting environment. In Workshop on the Theory and Practice of Provenance (TaPP), Cologne, Germany.

[24] Dionysios Logothetis, Soumyarupa De, and Kenneth Yocum. 2013. Scalable lineage capture for debugging disc analytics. In Proceedings of the 4th annual Symposium on Cloud Computing. ACM, 17.

[25] Boon Thau Loo and Joseph M Hellerstein. 2006. The design and implementation of declarative networks. Ph.D. Dissertation. University of California, Berkeley.

[26] Keith Ansel Marzullo. 1984. Maintaining the time in a distributed system: an example of a loosely-coupled distributed service (synchronization, fault-tolerance, debugging). (1984).

[27] Leonardo Murta, Vanessa Braganholo, Fernando Chirigati, David Koop, and Juliana Freire. 2014. noWorkflow: capturing and analyzing provenance of scripts. In International Provenance and Annotation Workshop. Springer, 71-83.

[28] Hyunjung Park, Robert Ikeda, and Jennifer Widom. 2011. Ramp: A system for capturing and tracing provenance in mapreduce workflows. (2011).

[29] Amritha Sampath and C Tripti. 2012. Synchronization in distributed systems. In Advances in Computing and Information Technology. Springer, 417-424.

[30] Ulrich Schmid. 2000. Orthogonal accuracy clock synchronization. Chicago fournal of Theoretical Computer Science 3, 2000 (2000), 3-77.

[31] Fred B Schneider. 1990. Implementing fault-tolerant services using the state machine approach: A tutorial. ACM Computing Surveys (CSUR) 22, 4 (1990), 299-319.

[32] Konstantin Shvachko, Hairong Kuang, Sanjay Radia, and Robert Chansler. 2010. The hadoop distributed file system. In Mass storage systems and technologies (MSST), 2010 IEEE 26th symposium on. IEEE, $1-10$.

[33] Benjamin H Sigelman, Luiz Andre Barroso, Mike Burrows, Pat Stephenson, Manoj Plakal, Donald Beaver, Saul Jaspan, and Chandan Shanbhag. 2010. Dapper, a large-scale distributed systems tracing infrastructure. Technical Report. Technical report, Google, Inc.

[34] Eno Thereska, Brandon Salmon, John Strunk, Matthew Wachs, Michael Abd-El-Malek, Julio Lopez, and Gregory R Ganger. 2006. Stardust: tracking activity in a distributed storage system. In ACM SIGMETRICS Performance Evaluation Review, Vol. 34. ACM, 3-14.

[35] Katherine Wolstencroft, Robert Haines, Donal Fellows, Alan Williams, David Withers, Stuart Owen, Stian Soiland-Reyes, Ian Dunlop, Aleksandra Nenadic, Paul Fisher, et al. 2013. The Taverna workflow suite: designing and executing workflows of Web Services on the desktop, web or in the cloud. Nucleic acids research 41, W1 (2013).

[36] Allison Woodruff and Michael Stonebraker. 1997. Supporting finegrained data lineage in a database visualization environment. In Data Engineering, 1997. Proceedings. 13th International Conference on. IEEE, 91-102. 
[37] Justin M Wozniak, Timothy G Armstrong, Michael Wilde, Daniel S Katz, Ewing Lusk, and Ian T Foster. 2013. Swift/t: Large-scale application composition via distributed-memory dataflow processing. In Cluster, Cloud and Grid Computing (CCGrid), 2013 13th IEEE/ACM International Symposium on. IEEE, 95-102.

[38] Matei Zaharia, Mosharaf Chowdhury, Michael J Franklin, Scott Shenker, and Ion Stoica. 2010. Spark: Cluster computing with working sets. HotCloud 10, 10-10 (2010).

[39] Xu Zhao, Yongle Zhang, David Lion, Muhammad Faizan Ullah, Yu Luo, Ding Yuan, and Michael Stumm. 2014. Iprof: A Non-intrusive Request Flow Profiler for Distributed Systems. In OSDI, Vol. 14. 629-644.

[40] Wenchao Zhou, Suyog Mapara, Yiqing Ren, Yang Li, Andreas Haeberlen, Zachary Ives, Boon Thau Loo, and Micah Sherr. 2012. Distributed time-aware provenance. In Proceedings of the VLDB Endowment, Vol. 6. VLDB Endowment, 49-60.

[41] Wenchao Zhou, Micah Sherr, Tao Tao, Xiaozhou Li, Boon Thau Loo, and Yun Mao. 2010. Efficient querying and maintenance of network provenance at internet-scale. In Proceedings of the 2010 ACM SIGMOD International Conference on Management of data. ACM, 615-626. 